Heliocentric zoning of the asteroid belt by aluminum-26 heating. R. E. Grimm;

\title{
$N 94-1$
} Department of Geology, Arizona State University, Tempe, AZ 85287-1404, and H. Y. McSween, Jr., Department of Geological Sciences, University of Tennessee, Knoxville, TN 37996-1410.

Variations in petrology among meteorites attest to a strong heating event early in solar system history, but the heat source has remain unresolved (e.g., 1). Aluminum-26 has been considered the most likely high-energy, short-lived radionuclide (half-life 0.72 million years) since the discovery of its decay product - excess $26 \mathrm{Mg}$ - in Allende CAIs (2). Furthermore, observation of relict $26 \mathrm{Mg}$ in an achondritic clast and in feldspars within ordinary chondrites (3, 4) provided strong evidence for live $26 \mathrm{Al}$ in meteorite parent bodies and not just in refractory nebular condensates. The inferred amount of $26 \mathrm{Al}$ is consistent with constraints on the thermal evolution of both ordinary (5) and carbonaceous (6) chondrite parent objects up to a few hundred kilometers in diameter.

Meteorites can constrain the early thermal evolution of their parent body locations, provided that a link can be established between asteroid spectrophotometric signature and meteorite class (7). Asteroid compositions are heliocentrically distributed (8): Objects thought to have experienced high metamorphic or even melting temperatures are located closer to the sun, whereas apparently unaltered or mildly heated asteroids are located farther away (9).

Heliocentric zoning could be the result of $26 \mathrm{Al}$ heating if the initial amount of the radionuclide incorporated into planetesimals was controlled by accretion time, which in turn varies with semimajor axis. Analytic expressions for planetary accretion (10) may be integrated to give the time $\tau$ required for a planetesimal to grow to a specified radius: $\tau \propto a^{n}$, where $n=1.5$ to 3 depending on the assumptions about variations in the surface density of the planetesimal swarm. Numerical simulations of planetesimal accretion at fixed semimajor axis (11) demonstrate that variations in accretion time among small planetesimals can be strongly nonlinear depending on the initial conditions and model assumptions. The general relationship with semimajor axis remains valid because it depends only on the initial orbital properties and distribution of the planesimal swarm.

In order to demonstrate the basic dependence of thermal evolution on semimajor axis, we parameterized accretion time across the asteroid belt according to $\tau \propto a^{n}$ and calculated the subsequent thermal history. Objects at a specified semimajor axis were assumed to have the same accretion time, regardless of size. We set the initial $26 \mathrm{Al}^{27} \mathrm{Al}$ ratio $=6 \times 10^{-5}(2)$ and treated $n$ and $\tau_{O}$ at $a_{O}=3 \mathrm{AU}$ as adjustable parameters. The thermal model (6) included temperature-dependent properties of ice and rock (CM chondrite analog) and the thermodynamic effects of phase transitions.

The principal observational constraints to be met are the inferred heliocentric distances for melting of chondritic silicates and of water ice within asteroids, taken to occur at temperatures of $1100^{\circ} \mathrm{C}$ and $0^{\circ} \mathrm{C}$, respectively. The position of the silicate melting isotherm is uncertain because of the controversy concerning the nature of $S$-asteroids and the origin of ordinary chondrites. One recent summary (9) considers S-types to be differentiated and divides the asteroids into igneous, metamorphic and primitive types with boundaries approximately at 2.7 and $3.4 \mathrm{AU}$. The boundary between igneous and metamorphic classes should therefore correspond roughly to the $1100^{\circ} \mathrm{C}$ isotherm. Ordinary chondrites are held to originate sunward of this boundary but from smaller objects that experienced greater heat loss and were unmelted. Evidence for hydrated silicates in some low-albedo asteroids $(12,13)$ is now taken to support the idea that aqueous alteration occurred by melting of ice and reaction with anhydrous silicates. Such asteroids are generally dominant around $3 \mathrm{AU}$, but objects farther out show decreasing spectral signatures of hydrated silicates, suggesting that liquid water was not present there. The isotherm for ice melting is the basis for the division between metamorphic and primitive asteroid types (9) at $3.4 \mathrm{AU}$.

Incorporation of ice into planetesimals can strongly affect thermal history (6) in that ice provides a thermal buffer that substitutes low-temperature aqueous alteration (as in carbonaceous chondrites) for high-temperature metamorphic recrystallization (as in ordinary and enstatite chondrites). The distribution of ice in the asteroid belt before the heating event is uncertain. 
Therefore we have performed two sets of thermal calculations: in the first (the anhydrous model) the thermal effects of ice were neglected; all model objects were assumed to be $90 \%$ rock and $10 \%$ voids. In the second set (the hydrous model) we explicitly introduced ice beyond $2.7 \mathrm{AU}$. Model objects beyond here have compositions of $60 \%$ rock, $30 \%$ ice, and $10 \%$ voids by volume.

The positions of the silicate and ice melting isotherms agree with observations for large diameters for the anhydrous model with $\tau_{0}=3.5$ million years and for the hydrous model with $\tau_{0}$ $=3.0$ million years (Fig. 1). A shorter accretion time is required for the hydrous model because a larger complement of $26 \mathrm{Al}$ is necessary to achieve the specified threshold temperatures when ice is present. Both of these times are upper limits because objects will radioactively heat during formation and reach limiting temperatures more rapidly than in our model where full-grown objects start cold. In both the anhydrous and hydrous models the best fit was achieved with $n=$ 3 , although $n=2.5$ gave comparable results if $\tau_{0}$ was increased slightly. The large value of $n$ is required to accommodate the strong variation in temperature over $<1 \mathrm{AU}$ and is in agreement with suggestions that the surface density of the planetesimal swarm varied as $a^{-1}$ to $a^{-3 / 2}(14$, 15). Such values of $n$ lead to variations in accretion time of several half-lives of $26 \mathrm{Al}$ across the asteroid belt (Fig. 1).

These calculations demonstrate that heliocentric zoning of the asteroid belt can be achieved by $26 \mathrm{Al}$ heating with reasonable assumptions about the variation of accretion time with semimajor axis (16). Although solar electromagnetic induction heating has gained favor with the recognition of a sun-centered heating pattern in the asteroid belt, that model is highly simplified and contains considerable uncertainties in parameter choices (17). We cannot reject this hypothesis with present data, but we favor $26 \mathrm{Al}$ heating because it can explain the observed data with fewer assumptions and because $26 \mathrm{Al}$ is known to have been present in significant quantities in newly accreted meteorite parent bodies (3).

References (1) Wood and Pellas, in The Sun in Time (Univ. of Arizona Press, Tucson, 1991) pp. 740-760. (2) Lee et al., GRL 3, 109-112 (1976). (3) Hutcheon et al., Nature 337, 238-241 (1989). (4) Zinner and Gopel, Meteoritics 27, 311-312 (1992). (5) Miyamoto et al., Proc. Lunar Planet. Sci. Conf. 12B, 1145-1152 (1981). (6) Grimm and McSween, Icarus 82, 244-280 (1989). (7) Gaffey and McCord, Space Sci. Rev. 21, 555-628 (1978). (8) Gradie and Tedesco, Nature 216, 1405-1407 (1982). (9) Bell et al., in Asteroids II (Univ. of Ariz. Press, Tucson, 1989) pp. 921-945. (10) Wetherill, Ann. Rev. Astron. Astrophys. 18, 77-113 (1980). (11) Wetherill and Stewart, Icarus 77, 330-357 (1989). (12) Lebofsky et al., Icarus 48, 453-459 (1981). (13) Jones et al., Icarus 88, 172-192 (1990). (14) Weidenschilling, Astrophys. Space. Sci. 51, 153-158 (1977). (15) Wetherill and Chapman, in Meteorites and the Early Solar System (Univ. of Ariz. Press, Tucson, 1988). (16) Grimm and McSween, Science, in press, 1993. (17) Sonett et al., Astrophys. Space Sci 7, 446-488 (1970).

Fig 1. Peak temperatures in model objects as functions of size and semimajor axis for hydrous and anhydrous models. Dependence of accretion time on semimajor axis described in text. Shaded bands mark major divisions of the asteroid belt based on spectrophotometric data (9).
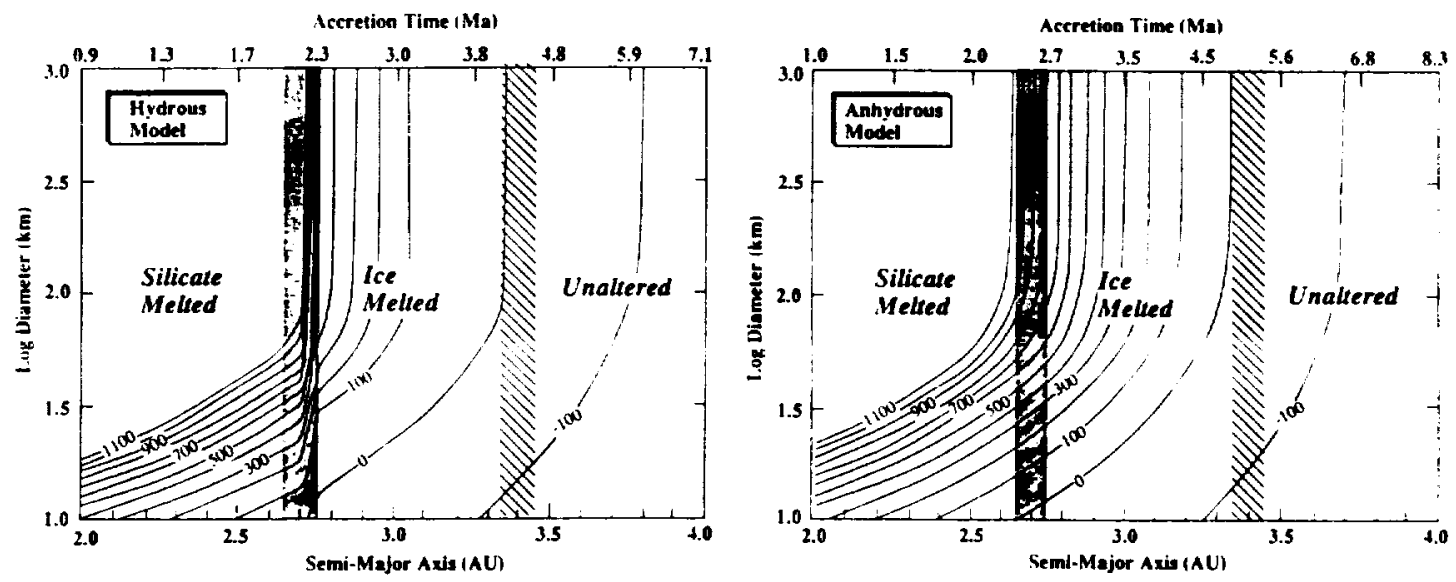